\title{
INCIDENCIA DEL DISEÑO EN PLANTA DE UNA PROTECCIÓN DE ESTRIBO SOBRE LAS EROSIONES RESULTANTES
}

\author{
Marcela L. Reynares, Mario I. Schreider, Graciela B. Scacchi \\ Facultad de Ingeniería y Ciencias Hídricas \\ Universidad Nacional del Litoral, Santa Fe, Argentina \\ reynares@fich1.unl.edu.ar
}

\begin{abstract}
Resumen: Las cubiertas flexibles son medidas de protección frecuentemente utilizadas para contrarrestar procesos erosivos locales que se desarrollan en sitios de puentes.

En este trabajo se analizan, de manera experimental, la incidencia que sobre las erosiones resultantes tienen la longitud de la protección (en el sentido de la corriente principal) y la extensión de la misma desde el extremo del estribo hacia la margen, y con su correspondiente modo de fijación al lecho. Se estudio también, la modificación que sufre la típica configuración 3D del flujo junto a un estribo como consecuencia de la presencia de la protección y el impacto que dicho proceso tiene sobre los mecanismos de erosión actuantes.

Los resultados muestran que el desarrollo longitudinal del sector erosionado se incrementó de acuerdo con la longitud que la protección tuvo en cada caso, afectando zonas del lecho no comprometidas en la situación sin protección. El ancho de la hoya de erosión no experimentó cambios significativos ante variaciones en las dimensiones del revestimiento. Protecciones de estribos largas promueven la superposición de mecanismos 3D, típicos en las cercanías del estribo, con fenómenos 2D, asociados a la contracción del flujo, cuya incidencia se incrementa hacia aguas abajo del mismo.
\end{abstract}

\section{INTRODUCCIÓN}

La colocación de protecciones flexibles junto a pilas y estribos son herramientas de la ingeniería cuya utilización ha tenido una creciente aceptación en los últimos tiempos, como medida para contrarrestar los procesos erosivos locales que se desarrollan en las inmediaciones de estas estructuras.

En general, en la literatura las dimensiones en planta de una protección se asocian a las erosiones esperables junto a un estribo no protegido o a la profundidad del flujo en las proximidades de la estructura. Sin embargo, se puede considerar que dicha erosión podría modificarse tanto en profundidad como en ubicación, por la misma presencia de la protección y aún más, que exista una relación entre las dimensiones de la protección y la configuración geométrica de la hoya de erosión resultante.

El presente estudio se enmarca en una investigación de mayor alcance sobre los procesos erosivos desarrollados en torno a estribos largos protegidos en valles de inundación, aspecto aún no resuelto por completo en la bibliografía especializada.

\section{MARCO CONCEPTUAL}

Los mecanismos de erosión actuantes junto a un estribo han sido ampliamente estudiados, tal como lo refleja la literatura específica (Melville y Coleman, 2000). La compleja estructura del escurrimiento responsable de dichos procesos tiene su origen en la alteración del flujo en su encuentro con el estribo. De la misma se distingue como mecanismo determinante del proceso, un vórtice principal, originado por la combinación de los flujos descendentes, junto a la cara de aguas arriba del estribo, y el de aproximación, desviado en las inmediaciones del mismo. Este vórtice, localizado junto al estribo es el de mayor potencial erosivo y en consecuencia el responsable de las zonas de más profundización. Su presencia induce a la formación de un vórtice secundario más débil, también de eje horizontal, ubicado a continuación del mismo. La separación del flujo en el borde del estribo da lugar a capas de corte inestable que se enrollan desencadenando los vórtices de estela, de eje vertical, cuya participación en el proceso erosivo es de menor importancia que los anteriores. Esta compleja configuración de flujo caracteriza al fenómeno erosivo como típicamente tridimensional. 
Las investigaciones realizadas para evaluar este tipo de erosiones han combinado modelos conceptuales de funcionamiento del proceso, herramientas de análisis dimensional y diseños experimentales, dando lugar a numerosas fórmulas de cálculo, que permiten evaluar la máxima profundidad de erosión, las que fueron recopiladas en Melville y Coleman (2000), May et al. (2002) y García (2008).

Para el caso de puentes aliviadores, que presenten estribos largos, Scacchi et al. (2005) demostró que las erosiones locales junto a este tipo de estructuras no pueden ser estudiadas a partir de las condiciones del flujo de aproximación, sino que responden a las condiciones iniciales del flujo junto al estribo y a la transferencia lateral de caudal a la hoya de socavación. Los autores incorporaron el ancho de la sección del puente como variable representativa del proceso de transferencia lateral de caudales, redefiniendo así, la aplicación de las ecuaciones de estribo largo a situaciones de puentes aliviadores.

La colocación de una protección alrededor de un estribo introduce una alteración en el proceso erosivo que, a la hora de analizar el mismo, pone en tela de juicio la validez de extrapolar a esta situación particular, las conclusiones obtenidas de los estudios correspondientes a un estribo no protegido.

Los estudios antecedentes disponibles abocados al diseño de protecciones en general han concentrado sus esfuerzos en la determinación del espesor mínimo requerido para asegurar la integridad de estos dispositivos (Richardson et al., 1995; Chiew, 1995; Przedwojski et al., 1995; Melville y Coleman, 2000; Lauchlan y Melville, 2001), y en menor medida las dimensiones en planta que debieran tener los mismos (Témez, 1988; Lagasse et al., 2001; Melville et al., 2006; Cardoso y Fael; 2009).

Resultan escasos los estudios que incluyen en su análisis la respuesta erosiva del lecho junto a la protección. Reynares et al. (2005) llevó a cabo una serie de experimentos de laboratorio tomando como base los resultados de Scacchi et al. (2005) e incorporando una protección rígida al pie de un estribo largo, con dos rugosidades distintas. Los resultados obtenidos indicaron que la presencia de la protección no mitiga el problema. Las erosiones se desarrollan alejadas del estribo, en sectores del cauce que antes no se veían afectados por la presencia de las mismas.

Melville et al. (2006) llevó a cabo un estudio experimental donde ensayaron protecciones constituidas por riprap y por bloques de concreto unidos con cables (CTB) en estribos ataludados intermedios, situados en valles de inundación de secciones compuestas. Los autores propusieron una expresión para determinar la extensión del delantal en función de la máxima erosión $\left(y_{e}\right)$. Con respecto a las erosiones, encontraron que la protección no reduce la magnitud de las mismas, sino que desvía la hoya lo suficientemente lejos del pie de estribo como para evitar daños en el mismo. Los revestimientos con CTB generaron hoyas más cercanas al estribo, en comparación con los de riprap, resultando fosas más profundas.

Morales et al. (2008) experimentaron con distintos ancho de protección $(W)$ en estribos ataludados, cortos $\mathrm{y} / \mathrm{o}$ intermedios. Encontraron que la forma inicial del estribo se modificó por la presencia de la protección lo que se tradujo en cambios en la localización y forma de la hoya de socavación resultante. Cuando el ancho de la protección fue mayor a la profundidad de erosión $\left(y_{e}\right)$-para la situación no protegida-, la hoya se alejó del estribo pero no se redujo en profundidad. Las máximas erosiones disminuyeron considerablemente en aquellas oportunidades donde $W$ superó las 1.5 veces el tirante medio del flujo $\left(h_{a}\right)$.

El presente trabajo tiene por objeto estudiar los procesos erosivos junto a un estribo protegido con bloques de concreto adheridos a geotextil. En particular se propone analizar:

1. La incidencia que sobre las erosiones resultantes tiene la extensión de la protección definida desde el extremo del estribo hacia la margen en la cual se inserta y el modo de fijación al lecho asociado a la misma.

2. La incidencia que la longitud de la protección, en la dirección principal del escurrimiento, tiene con respecto a :

a) Magnitud y ubicación de la máxima profundidad de erosión.

b) Forma en planta y volumen del sector erosionado junto a la protección.

3. Las modificaciones que sufre la típica configuración 3D del flujo junto al estribo, como consecuencia de la presencia de la protección, identificando la existencia de zonas de flujo junto a la misma con características bidimensionales, semejantes a una contracción gradual. 


\section{DISEÑO EXPERIMENTAL}

La metodología de análisis llevada a cabo fue de tipo experimental. La misma constó de dos etapas, en la cuales se desarrollaron ensayos de laboratorio en condiciones controladas, acordes a los objetivos perseguidos. Los dispositivos utilizados y las condiciones adoptadas en cada etapa se describen a continuación.

\section{Etapa I}

Los experimentos correspondientes a esta etapa del estudio se llevaron a cabo en un canal ubicado en la Nave I del Laboratorio de Hidráulica de la Facultad de Ingeniería y Ciencias Hídricas $(\mathrm{FICH})$, de $18.50 \mathrm{~m}$ de largo, $7.65 \mathrm{~m}$ de ancho y $0.90 \mathrm{~m}$ de profundidad (Figura 1 ).

El canal posee un tramo de ingreso a fondo fijo de $3.50 \mathrm{~m}$ de longitud, luego del cual se desarrolla en todo el ancho del canal un sector con lecho móvil de $11 \mathrm{~m}$ de longitud y $0.60 \mathrm{~m}$ de espesor, constituido por arena uniforme con un d50 de $0.001 \mathrm{~m}$. El tramo final está conformado por otro sector rígido de $3.50 \mathrm{~m}$ de longitud.

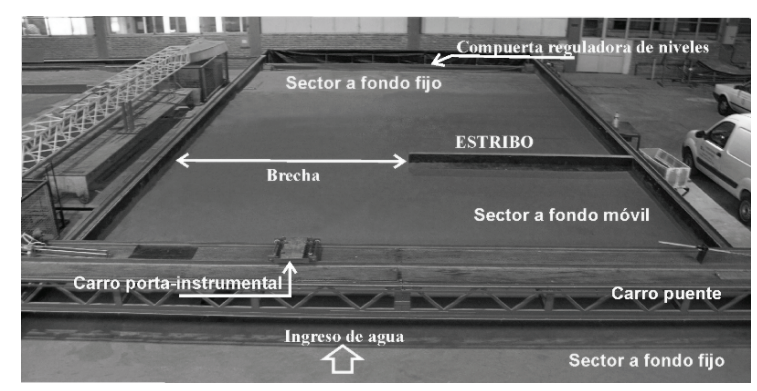

Figura 1. Dispositivo experimental de la Etapa I

A $8.50 \mathrm{~m}$ aguas abajo de la sección de entrada, en el sector a fondo móvil, se encuentra materializado un cierre parcial de 3.65 metros de extensión, con origen en la margen derecha del cuenco, configurando así un estribo prismático de pared vertical y $0.12 \mathrm{~m}$ de espesor.

Junto al pie del estribo se colocó una protección del tipo de Bloques de Concreto Adheridos a Geotextil (BCAG), los cuales tienen una sección cuadrada de $0.027 \mathrm{~m}$ de lado y $0.012 \mathrm{~m}$ de espesor. Los prismas se encuentran separados entre sí una distancia de $0.002 \mathrm{~m}$ (Figura 2). La altura del bloque fue determinada mediante la expresión de Pilarczyk (1997), de acuerdo a las condiciones hidráulicas reproducidas en la serie experimental.

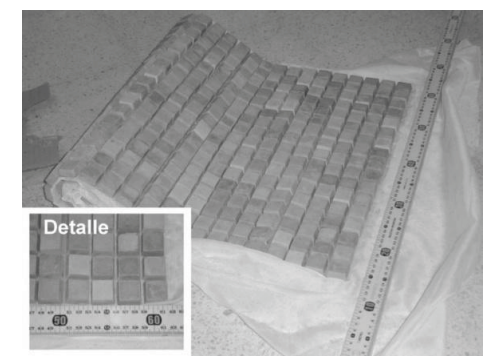

Figura 2. Protección de Bloques de Concreto Adherido a Geotextil (BCAG)

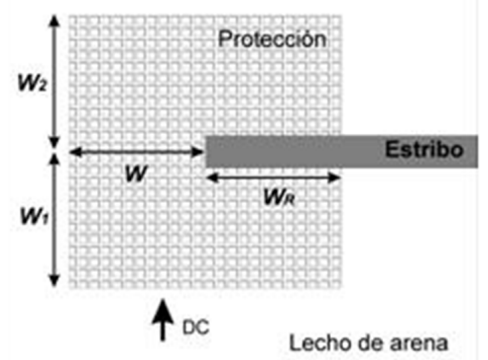

Figura 3. Esquema de las dimensiones de la protección

El diseño en planta de la protección utilizada se caracterizó por las siguientes dimensiones (Figura 3):

1. W: distancia medida desde el extremo del estribo en sentido transversal a la corriente. La misma se mantuvo constante en todas las series de experimentos.

2. $W_{1}$ : longitud de la protección en dirección hacia aguas arriba, medida desde el eje del estribo.

3. $W_{2}$ : longitud de la protección en dirección hacia aguas abajo, medida desde el eje del estribo.

4. $W_{R}$ : extensión de protección medida desde el extremo del estribo en dirección hacia la margen donde éste se inserta.

En esta etapa se realizaron un (1) ensayo de referencia y dos (2) series de experimentos, con objetivos específicos cada una de ellas.

Todos los ensayos llevados a cabo tuvieron un caudal total constante de $0.144 \mathrm{~m}^{3} / \mathrm{s}$, un tirante (calado) del flujo de aproximación de $0.12 \mathrm{~m}$ y fueron ejecutados en condiciones de agua clara, bajo condiciones próximas a la de iniciación de movimiento en la sección de emplazamiento del puente. La duración de todos los experimentos fue de 24 horas. Este tiempo ha sido considerado suficiente para alcanzar un desarrollo de la máxima profundidad de erosión local próximo al 
$80 \%$, respecto del valor final de equilibrio, tal como fue demostrado por Schreider et al. (1998). Asimismo a los efectos de evaluar la incidencia sobre las erosiones resultantes, la comparación entre ensayos de igual duración permite arribar a conclusiones que responden a los objetivos que el trabajo persigue.

\section{Ensayo de Referencia}

En primer lugar se llevó a cabo un ensayo patrón $\left(E_{R}\right)$, en el cual se dejó desarrollar la hoya de erosión al pie del estribo, con el objetivo de contrastar los resultados obtenidos en el mismo con los de las Series 1 y 2 , en las que el estribo estuvo protegido. Las condiciones hidráulicas de este ensayo fueron las señaladas con anterioridad.

\section{Serie 1}

En esta serie se desarrollaron cuatro (4) experimentos con el propósito de analizar las consecuencias que sobre las erosiones y la estabilidad de la manta tiene el modo en que se fija el extremo de la protección próximo a la margen.

En todos los ensayos las dimensiones de la manta $\left(W=W_{1}=W_{2}=0.50 \mathrm{~m}\right)$ se mantuvieron constantes. Las características de cada uno de ellos en cuanto a la extensión $W_{R}$ y el modo de anclaje de la misma, se describen en Tabla 1.

\section{Serie 2}

En esta serie se llevaron a cabo cuatro (4) ensayos, con el propósito de evaluar la incidencia del largo de la protección $\left(W_{1}+W_{2}\right)$ sobre la máxima profundidad de erosión, esto es, su ubicación, área y volumen erosionados. En todos los ensayos $W_{1}$ fue igual a $W_{2}$ de modo de establecer una protección simétrica respecto del eje del estribo. El ancho $W$ se mantuvo constante e igual a $0.50 \mathrm{~m}$, al igual que la extensión $W_{R}$ la cual fue de $0.50 \mathrm{~m}$ y estuvo unida al resto del revestimiento por medio de una costura. Las longitudes adoptadas para cada ensayo se presentan en la Tabla 2.

En la totalidad de los experimentos de esta etapa se midió el pelo de agua (superficie libre) mediante limnímetro provisto de punta y vernier, y nivel óptico de precisión.

Finalizado cada experimento, el canal fue desagotado por medio de una serie de drenes ubicados en el fondo del mismo. El número y disposición de los drenes aseguró un vaciado lento y gradual, evitando cualquier tipo de alteraciones en el lecho resultante. Una vez drenado el mismo, se realizó un relevamiento de detalle de la zona erosionada. Para ello se empleó un distanciómetro láser, con una precisión inferior al milímetro, desde un carro porta-instrumental (Figura 1).

\section{Etapa II}

Los experimentos correspondientes a esta etapa se desarrollaron en un canal ubicado en la Nave II del Laboratorio de Hidráulica de la Facultad de Ingeniería y Ciencias Hídricas (FICH), de $60 \mathrm{~m}$ de largo, $1.50 \mathrm{~m}$ de ancho y $1.00 \mathrm{~m}$ de profundidad (Figuras $4 a$ y $4 b$ ).

\begin{tabular}{|c|c|c|}
\hline Ensayo $N^{o}$ & Descripción del Ensayo & Esquema \\
\hline $\mathrm{E}_{\mathrm{S} 1-1}$ & $\begin{array}{c}W=W_{1}=W_{2}=0.50 \mathrm{~m} \quad W_{R}=0 \\
\text { Manta simplemente apoyada }\end{array}$ & \\
\hline $\mathrm{E}_{\mathrm{S} 1-2}$ & $\begin{array}{c}W=W_{l}=W_{2}=0.50 \mathrm{~m} \quad W_{R}=0 \\
\text { Protección anclada con } \\
\text { Zunchos* de alambres }\end{array}$ & \\
\hline$E_{\mathrm{S} 1-3}$ & $\begin{array}{c}W=W_{l}=W_{2}=0.50 \mathrm{~m} \quad W_{R}=0.50 \mathrm{~m} \\
\text { Protección extendida hacia la margen } \\
\text { con mantas geotextil superpuestas } \\
\text { simplemente apoyadas }\end{array}$ & \\
\hline $\mathrm{E}_{\mathrm{S} 1-4}$ & $\begin{array}{c}W=W_{l}=W_{2}=0.50 \mathrm{~m} \quad W_{R}=0.50 \mathrm{~m} \\
\text { Protección extendida hacia la margen } \\
\text { Con mantas de geotextil superpuestas cocidas }\end{array}$ & \\
\hline
\end{tabular}

Tabla 1. Descripción de los ensayos realizados en la Serie 1 


\begin{tabular}{|c|c|c|}
\hline Ensayo $N^{\circ}$ & Descripción del Ensayo & Esquema \\
\hline $\mathrm{E}_{\mathrm{S} 2-1}$ & $\begin{array}{c}W=0.50 \mathrm{~m} \\
W_{R}=0.50 \mathrm{~m} \\
W_{1}=W_{2}=0.20 \mathrm{~m}\end{array}$ & \\
\hline $\mathrm{E}_{\mathrm{S} 2-2}$ & $\begin{array}{c}W=0.50 \mathrm{~m} \\
W_{R}=0.50 \mathrm{~m} \\
W_{1}=W_{2}=0.50 \mathrm{~m}\end{array}$ & \\
\hline $\mathrm{E}_{\mathrm{S} 2-3}$ & $\begin{array}{c}W=0.50 \mathrm{~m} \\
W_{R}=0.50 \mathrm{~m} \\
W_{1}=W_{2}=0.72 \mathrm{~m}\end{array}$ & \\
\hline $\mathrm{E}_{\mathrm{S} 2-4}$ & $\begin{array}{c}W=0.50 \mathrm{~m} \\
W_{R}=0.50 \mathrm{~m} \\
W_{1}=W_{2}=0.95 \mathrm{~m}\end{array}$ & \\
\hline
\end{tabular}

Tabla 2. Descripción de los ensayos realizados en la Serie 2

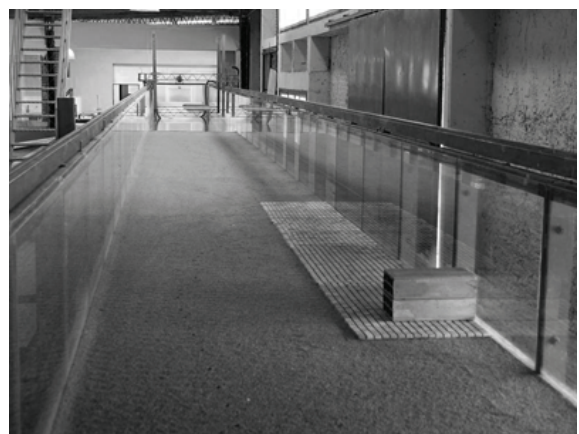

(a)

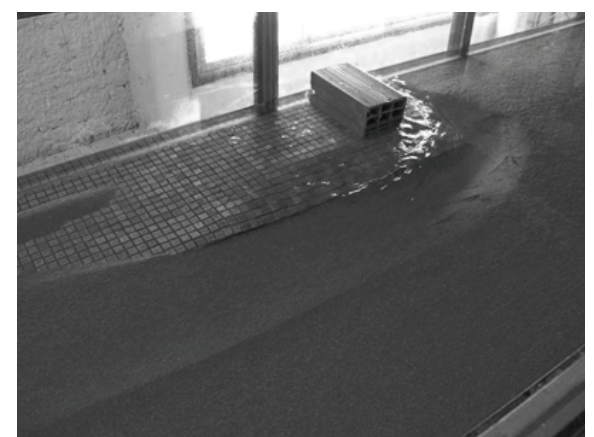

(b)

Figura 4. Vistas del dispositivo de ensayo de la Etapa II

Los $30 \mathrm{~m}$ finales del canal poseen paredes de vidrio, lo cual permite la visualización de los fenómenos que se representan. En dicho sector se conformó, todo a lo ancho del canal, un lecho erosionable de $20 \mathrm{~m}$ de longitud y $0.30 \mathrm{~m}$ de espesor, constituido por arena uniforme de diámetro medio $0.001 \mathrm{~m}$. En la sección media del tramo a fondo móvil se materializó, junto a la margen derecha, un estribo prismático de $0.32 \mathrm{~m}$ de longitud (en sentido transversal a la 
corriente principal) y $0.15 \mathrm{~m}$ de ancho (en sentido de la corriente principal). Alrededor del mismo se colocó una protección de lecho de iguales características constructivas que las utilizadas en el canal de la Etapa I, pero con las siguientes dimensiones: $W=0,20 \mathrm{~m}, W_{1}=0,20$ $\mathrm{m}, W_{2}=1,60 \mathrm{~m}$ y $W_{R}=0,32 \mathrm{~m}$.

Las condiciones hidráulicas definidas para el ensayo fueron las siguientes:

- Caudal $Q=0,048 \mathrm{~m}^{3} / \mathrm{s}$.

- Tirante $h=0,125 \mathrm{~m}$.

- Velocidad media $V=0,256 \mathrm{~m} / \mathrm{s}$.

La velocidad media del escurrimiento, en relación con la condición crítica de iniciación de movimiento del sedimento del lecho, determinó condiciones de agua clara en el flujo de la sección contraía $\left(V / V_{c}=0,70\right)$, reproduciéndose así condiciones hidráulicas semejantes a las establecidas en los ensayos realizados en la Etapa I.

El experimento tuvo una duración de cinco días, tiempo para el cual se logró una buena aproximación de los fenómenos bidimensionales típicos de erosiones por contracción gradual, alcanzándose a su vez un desarrollo mayor en las erosiones locales.

Durante el desarrollo experimental se midieron velocidades puntuales en diferentes secciones transversales ubicadas a lo largo de la protección (Figura 4a). Dicho registro tuvo lugar en dos momentos diferentes a lo largo del tiempo que duró el ensayo.

- Dentro de las 4 horas de iniciado el experimento. Esto es, con un grado de desarrollo de la erosión relativamente pequeño.

- Al cabo de 48 - 50 horas de iniciado el experimento.

Las profundidades del escurrimiento en las verticales de medición y las velocidades puntuales se midieron con velocímetro acústico doppler (ADV), el cual fue posicionado desde un carro porta instrumental que permite movimiento en las tres direcciones principales.

Luego de cada experimento, se desagotó el canal y se realizó un relevamiento de detalle del lecho en la zona erosionada, mediante metodologías semejantes a las descritas en la Etapa I.

\section{ANÁLISIS DE RESULTADOS}

Los resultados alcanzados se analizan en el contexto determinado por los objetivos propuestos en este trabajo.
Incidencia que tiene la extensión $W_{R}$ y correspondiente modo de fijación al lecho, sobre las erosiones resultantes

En primer lugar se presentan los parámetros característicos más sobresalientes que se observaron en el ensayo de referencia, $E_{R}$ :

- Profundidad Máxima $y_{e}=0,293 \mathrm{~m}$.

- Área superficial erosionada $A_{e}=1,798$ $\mathrm{m}^{2}$.

- Volumen de sedimento erosionado $V_{e}=$ $0,108 \mathrm{~m}^{3}$.

La hoya de erosión desarrollada junto al estribo se caracterizó por tener forma cónica, coincidente con la típica que se reporta en la mayoría de los estudios de erosión local junto a estribos de puentes sin protección. La máxima profundidad del lecho se localizó junto al vértice del estribo perteneciente a su cara de aguas arriba (Figura 5).

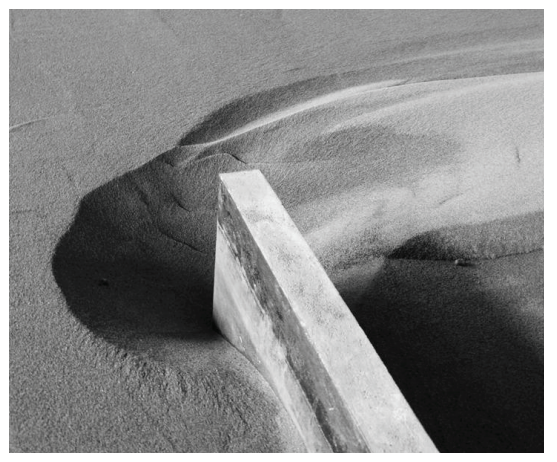

Figura 5. Hoya de erosión resultante en el ensayo $E_{R}$

El primer experimento realizado para la situación de estribo protegido correspondió a la situación $W_{R}=0,00 \mathrm{~m}$, en el que la protección fue simplemente apoyada sobre el lecho, sin ningún tipo de anclaje $\left(E_{S 1-1}\right)$. Como resultado se obtuvo un corrimiento de la protección (Figura 6a). Esto dio lugar a dos procesos erosivos superpuestos. En respuesta a ello se pueden identificar dos sectores de erosión bien diferenciados. Uno de pequeñas dimensiones, junto al estribo, y otro con profundizaciones más importantes, localizado junto al lado externo de la protección. La profundidad máxima de la hoya aquí conformada fue de $0.16 \mathrm{~m}$, sustancialmente menor a la medida en el ensayo de referencia $\left(E_{R}\right)$. 


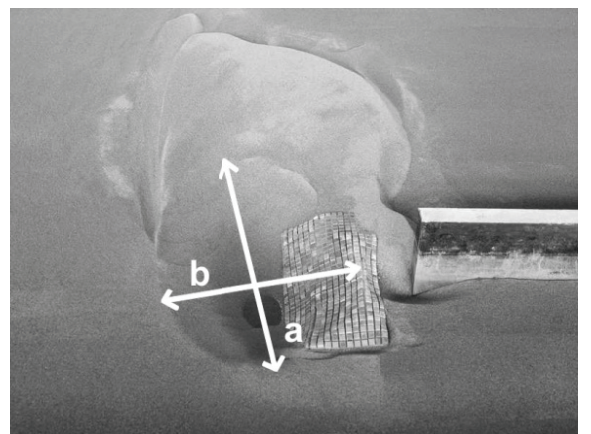

(a)

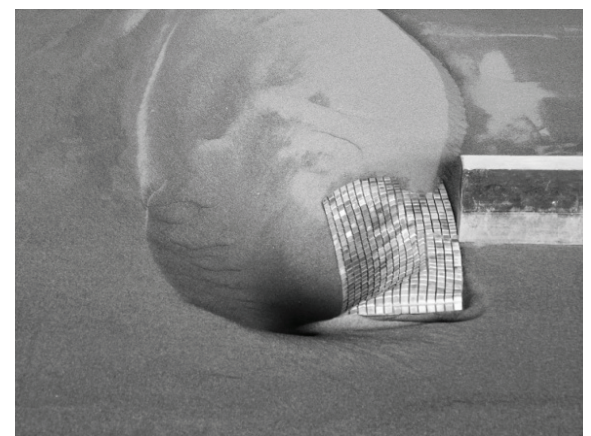

(b)

Figura 6. Fondo resultante en los ensayos $E_{S 1-1}$ y $E_{S 1-2}$

\begin{tabular}{|c|c|c|c|c|c|c|c|c|c|c|}
\hline $\begin{array}{l}\text { Ensayo } \\
\text { No. }\end{array}$ & $\begin{array}{c}W_{1}=W_{2} \\
{[\mathrm{~m}]}\end{array}$ & $\begin{array}{l}W_{R} \\
{[\mathrm{~m}]}\end{array}$ & $\begin{array}{l}y_{e} \\
{[\mathrm{~m}]}\end{array}$ & $\begin{array}{c}\text { Ancho } \\
{[\mathrm{m}]}\end{array}$ & $\begin{array}{c}\text { Área superficial } \\
{\left[\mathrm{m}^{2}\right]}\end{array}$ & $\begin{array}{c}\text { Volumen } \\
{\left[\mathrm{m}^{3}\right]}\end{array}$ & $\begin{array}{c}a \\
{[\mathrm{~m}]}\end{array}$ & $\begin{array}{c}b \\
{[\mathrm{~m}]}\end{array}$ & $F=a / b$ & Detalle \\
\hline$E_{R}$ & 0.00 & 0.00 & 0.293 & 1.027 & 1.798 & 0.108 & 1.52 & 1.27 & 1.21 & sin manta \\
\hline \multirow[t]{2}{*}{$E_{S 1-1}$} & \multirow[t]{2}{*}{0.50} & \multirow[t]{2}{*}{0.00} & & 0.530 & & - & 0.54 & 0.53 & 1.02 & \multirow[t]{2}{*}{ colapsada } \\
\hline & & & 0.090 & 0.750 & - & - & 1.64 & 0.75 & 2.18 & \\
\hline$E_{S 1-2}$ & 0.50 & 0.00 & 0.306 & 1.010 & 1.936 & 0.163 & 1.84 & 1.07 & 1.71 & anclada \\
\hline$E_{S 1-3}$ & 0.50 & 0.50 & 0.287 & 1.130 & 1.901 & 0.142 & 2.11 & 1.06 & 2.00 & solapada \\
\hline$E_{S 1-4}$ & 0.50 & 0.50 & 0.319 & 1.050 & 1.715 & 0.165 & 2.16 & 1.04 & 2.08 & cocida \\
\hline
\end{tabular}

Tabla 3. Descripción de los ensayos realizados en la Serie 1

El corrimiento de la protección, con la consecuente formación de una hoya de erosión local junto al estribo, puede ser el factor determinante de la erosión relativamente pequeña en el sector de mayores profundizaciones (Tabla 3).

Con el objeto de analizar la forma en planta del sector erosionado se definió el coeficiente de forma $F$, el cual se obtiene mediante la relación entre sus ejes paralelos a la dirección del flujo de aproximación y transversal al mismo, (a) y (b) respectivamente, esto es $F=a / b$ (Figura 6a). En el ensayo de referencia $\left(E_{R}\right)$, dicho coeficiente adquirió un valor de 1.21 , mientras que para las dos hoyas formadas en el ensayo $E_{S 1-1}$ fue:

- $F_{\text {hoya menor }}=1,02$

- $F_{\text {hoya mayor }}=2,18$

El valor de $F$ obtenido para la hoya desarrollada inmediatamente junto al estribo se corresponde con su forma cónica, típica de los procesos de erosión local en estribos sin protección. Cuando ésta estuvo presente, el desarrollo en planta del sector erosionado junto a la misma se tradujo en valores de $F$ que prácticamente duplicaron al anterior. El mayor desarrollo longitudinal de la hoya de socavación proporciona un primer indicio de la influencia que la presencia de la protección tiene sobre las características de los procesos de erosión intervinientes.
En el ensayo $E_{S 1-2}$ (Figura 6b) la protección fue anclada al lecho, evitándose así el corrimiento de la misma. En esta situación las erosiones finales resultaron sustancialmente mayores que las del ensayo $E_{S 1-1}$, similares a las de $E_{R}$ (Tabla 3). La ubicación de la máxima erosión se desplazó hacia aguas arriba. La forma en planta del sector erosionado fue más alargada respecto del ensayo de referencia, lo cual se tradujo en un valor de $F$ un $42 \%$ mayor al obtenido para $E_{S 1-1}$ (Tabla 3).

En los ensayos $E_{S 1-3}$ y $E_{S 1-4}$ se incorporó un tramo de protección hacia la margen, con una extensión $W_{R}=0,50 \mathrm{~m}$ con el objetivo de que el peso actúe como anclaje del conjunto. En el primer caso $\left(E_{S 1-3}\right)$ (Figura $\left.7 a\right)$ la manta fue simplemente apoyada permitiendo una superposición (solape) sobre el geotextil de la protección previamente colocada. Si bien la cubierta se desplazó de su posición original, la presencia del geotextil evitó la fuga de material en ese sector (Figura 7a). En el transcurso del experimento se desarrolló una profundidad máxima de erosión semejante a la del ensayo $E_{R}$. El ancho, el área superficial y el volumen de la hoya resultaron similares a los observados en el ensayo $E_{S 1-2}$ (Tabla 3), aunque la misma fue ligeramente más elongada. 


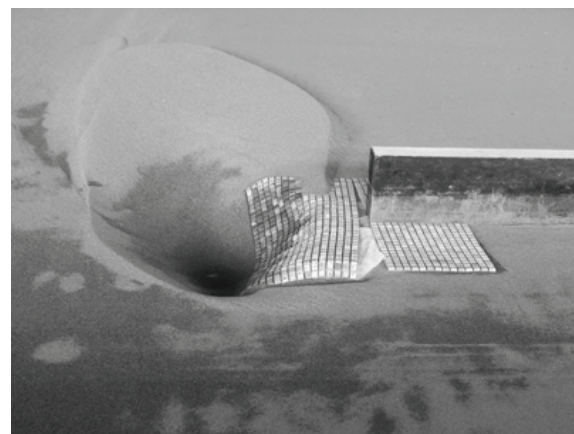

(a)

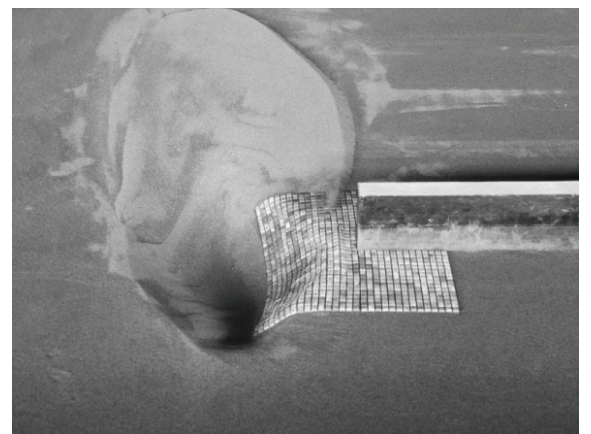

(b)

Figura 7. Fondo resultante en los ensayos $E_{S 1-3}$ y $E_{S 1-4}$

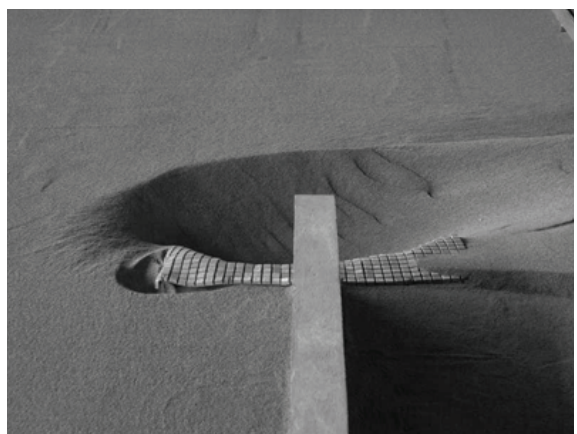

(a)

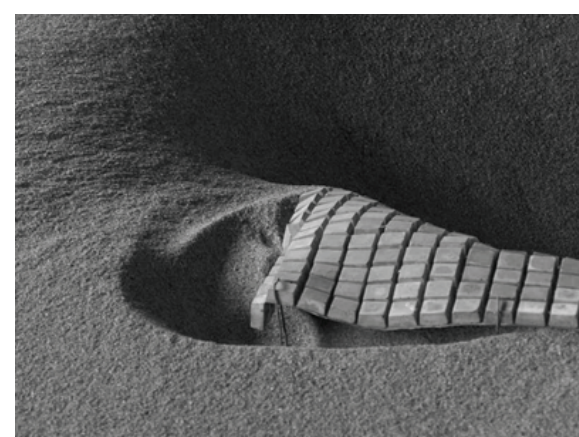

(b)

Figura 8. Anclaje expuesto en la zona del remate de aguas arriba del $E_{S 1-2}$

En el ensayo $E_{S 1-4}$ las mantas fueron cocidas, con el fin de evitar la separación de las mismas. La configuración final del lecho erosionado y la protección se presentan en la Figura $7 \mathrm{~b}$. Los valores característicos de profundidad, ancho, área superficial, volumen y factor de forma, resultaron semejantes a los de los ensayos precedentes $E_{S 1-2}$ y $E_{S 1-3}$, no observándose corrimientos de la protección.

De los resultados obtenidos en esta instancia del estudio se concluye que la incorporación de una longitud $W_{R}$ (Figura 3) a la protección del lecho junto al estribo, no provoca variaciones significativas en las erosiones resultantes, siempre que no se verifiquen desplazamientos de la manta por un inadecuado anclaje, lo que puede dar lugar a nuevos procesos erosivos en la adyacencia del estribo, como los que se observan en las Figuras $8 \mathrm{a}$ y $8 \mathrm{~b}$ correspondiente al Ensayo $E_{S 1-2}$ En función de dichas evidencias se consideró conveniente la incorporación de un tramo adicional de longitud $W_{R}$ que evite la aparición de posibles puntos fusibles, en los que los anclajes queden expuestos dándose lugar de este modo a mecanismos adicionales que puedan interferir con los objetivos de los ensayos planificados para la Serie 2. En consecuencia, se adoptó un valor de $W_{R}=0.50 \mathrm{~m}$ y una configuración de mantas cocidas entre sí, características que se mantuvieron constantes para todos los experimentos de la Serie 2.

Incidencia del desarrollo de la protección en sentido longitudinal de la corriente sobre la profundidad, forma en planta y volumen de la hoya de erosión

En esta instancia del estudio se ejecutaron cuatro experimentos de laboratorio, identificando a cada uno de ellos con el valor de $W_{1}$ y $W_{2}$ (Figura 3) que se le dio a la protección colocada junto al estribo. Estas dimensiones, como así también los principales parámetros que definieron al sector erosionado resultante se presentan en la Tabla 4.

El análisis de resultados encontrados se presenta sobre la base de los cuatro experimentos mencionados, atendiendo a los objetivos enunciados en segundo lugar. Esto es, evaluando la respuesta de la máxima profundidad de erosión, volumen y área erosionada, de acuerdo a la longitud que tuvo la protección $\left(W_{1}, W_{2}\right)$. 


\begin{tabular}{cccccccccc}
\hline $\begin{array}{c}\text { Ensayo } \\
\text { No. }\end{array}$ & $\begin{array}{c}W=W_{R} \\
{[\mathrm{~m}]}\end{array}$ & $\begin{array}{c}W_{1}=W_{2} \\
{[\mathrm{~m}]}\end{array}$ & $\begin{array}{c}\text { ye } \\
{[\mathrm{m}]}\end{array}$ & $\begin{array}{c}\text { ancho } \\
{[\mathrm{m}]}\end{array}$ & $\begin{array}{c}\text { Área superficial } \\
{\left[\mathrm{m}^{2}\right]}\end{array}$ & $\begin{array}{c}\text { volumen } \\
{\left[\mathrm{m}^{3}\right]}\end{array}$ & $\begin{array}{c}a \\
{[\mathrm{~m}]}\end{array}$ & $\begin{array}{c}b \\
{[\mathrm{~m}]}\end{array}$ & $\begin{array}{c}F=a / b \\
\text { ER }\end{array}$ \\
\hline 0.00 & 0.00 & 0.293 & 1.027 & 1.798 & 0.108 & 1.52 & 1.27 & 1.21 \\
$E_{S 1-2}$ & 0.50 & 0.20 & 0.298 & 1.000 & 2.456 & 0.189 & 2.40 & 1.30 & 1.85 \\
$E_{S 2-2}$ & 0.50 & 0.50 & 0.306 & 1.063 & 1.715 & 0.165 & 2.16 & 1.04 & 2.08 \\
$E_{S 2-3}$ & 0.50 & 0.72 & 0.292 & 1.100 & 1.796 & 0.153 & 2.13 & 1.05 & 2.04 \\
$E_{S 2-4}$ & 0.50 & 0.95 & 0.279 & 1.030 & 2.043 & 0.168 & 2.73 & 1.07 & 2.55 \\
\hline
\end{tabular}

Tabla 4. Descripción de los ensayos realizados en la Serie 2

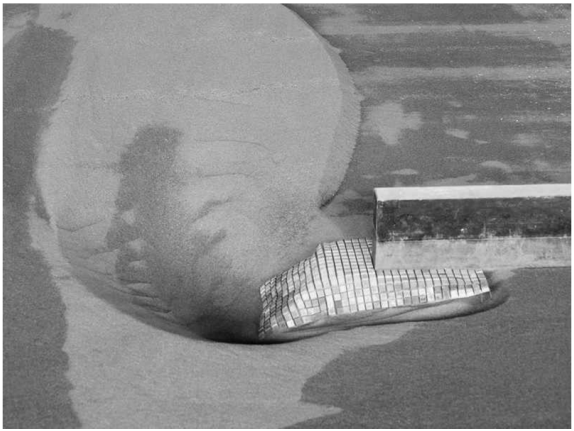

(a)

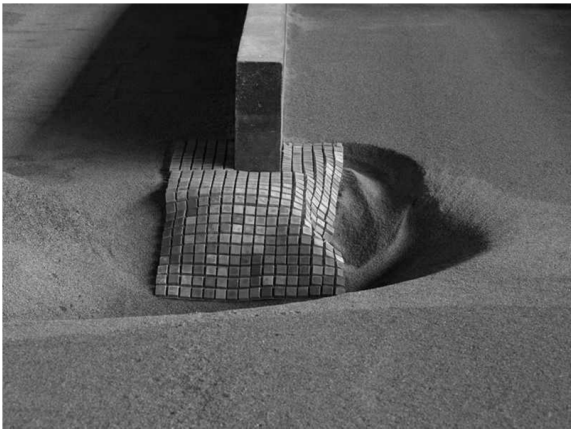

(b)

Figura 9. Fondo resultante en el ensayo $E_{S 2-1}$

La configuración del lecho en las inmediaciones de la protección que resultó del ensayo $E_{S 1-2}$ se presenta en la Figura 9. Tal como se puede observar en dicha figura la protección se ha deformado tanto en el sentido longitudinal como transversal a la corriente principal, encontrándose las mayores alteraciones en el extremo de aguas arriba del estribo (Figura 9b). La profundidad de erosión máxima fue semejante a la del ensayo $E_{R}$ (Tabla 4 ), pero localizada en un perfil transversal distante $0.10 \mathrm{~m}$ del eje del estribo, en dirección hacia aguas abajo, y desplazada $0.60 \mathrm{~m}$ hacia el centro del canal.

En las Figuras 10a, 10b y 10 c se muestran las configuraciones finales del lecho correspondiente a los ensayos $E_{S 2-2}, E_{S 2-3}$ y $E_{S 2-4}$ respectivamente. Los aspectos más importantes que surgieron de tales experiencias se resumen a continuación:

- Deformación de la protección.

A diferencia de lo ocurrido en el ensayo $E_{S 2-1}$ (protección de menor longitud) no se observaron deformaciones sobre el extremo de aguas arriba de la protección (Figura 10). Tales deformaciones se concentraron sobre el extremo lateral, opuesto al estribo, paralelo a la dirección longitudinal del flujo, involucrando una parte significa- tiva del talud derecho de la hoya de erosión resultante.

- Profundidad máxima de erosión:.

Los cuatro ensayos referidos presentaron profundidades máximas de erosión semejantes y muy próximas al valor testigo del ensayo $E_{R}$ (Tabla 4 ).

- Ubicación de la máxima profundidad de erosión.

En todos los experimentos la máxima profundidad se ubicó en las inmediaciones del eje del estribo pero desplazada hacia el centro de la sección una distancia aproximada de $0.60 \mathrm{~m}$, debido a la presencia de la protección.

- Forma en planta de la hoya de erosión. El desarrollo longitudinal del sector erosionado acompañó a la longitud dada a la protección en cada caso (Figura 10) sin cambios significativos en el ancho de la hoya correspondiente (Tabla 4). Esta situación se presenta a modo de ejemplo en la Figura 11, en la que se han superpuesto el contorno superficial de la hoya de erosión relevada al final del ensayo de referencia $\left(E_{R}\right)$ y los correspondientes a los ensayos $E_{S 2-2}$ y $E_{S 2-4}$. 


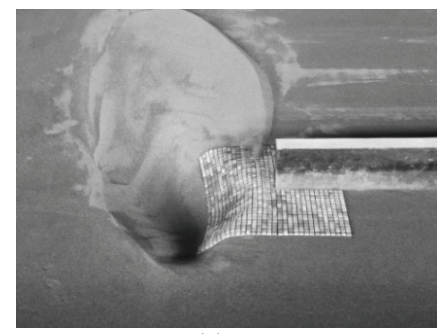

(a)

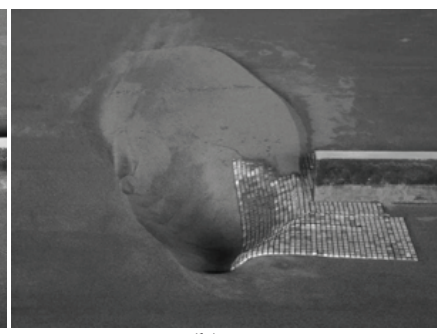

(b)

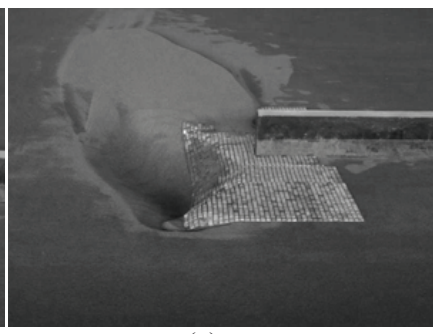

(c)

Figura 10. Fondo resultante en los ensayos $E_{S 2-2}, E_{S 2-3}$ y $E_{S 2-4}$

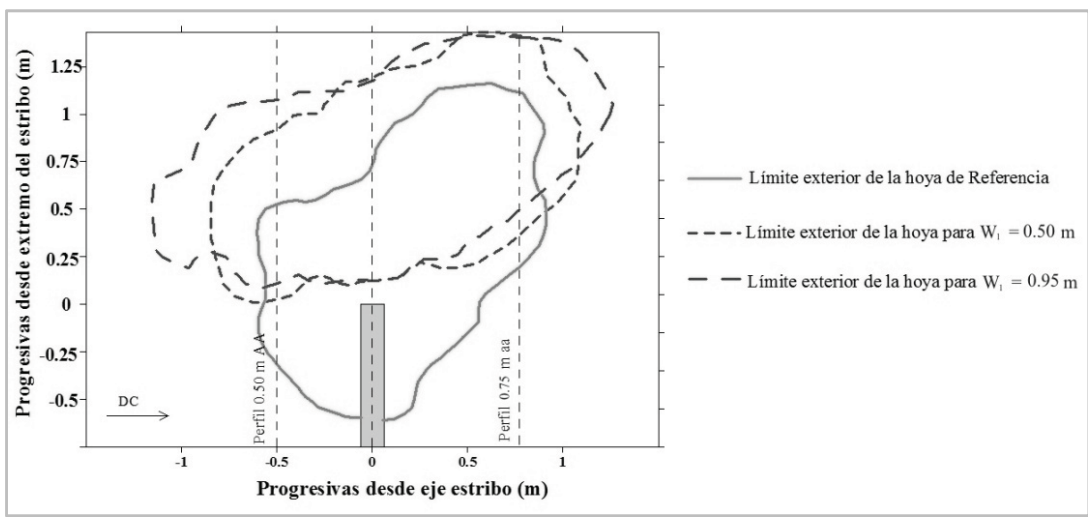

Figura 11. Contorno de las hoyas de erosión con $W_{1}=0 \mathrm{~m}, W_{1}=0.50 \mathrm{~m}$ y $W_{1}=0.95 \mathrm{~m}$

Con el fin de cuantificar la situación expuesta se han calculado los coeficientes de forma " $F$ ", definido con anterioridad, para la totalidad de los experimentos realizados en esta instancia (Tabla 4). El valor creciente de $F$ con el incremento de la longitud de la protección demuestra la mayor elongación del área erosionada.

Con el fin de evidenciar la influencia que la longitud de la protección tiene sobre las erosiones resultantes se han comparado perfiles transversales relevados en cada uno de los experimentos, correspondiente a tres ubicaciones diferentes $(0.50 \mathrm{~m}$ aguas arriba del eje del estribo, sobre el eje del estribo y $0.75 \mathrm{~m}$ aguas abajo del eje del estribo).

En la Figura 12 se presentan los datos medidos en la sección transversal a la dirección de la corriente, ubicada $0.50 \mathrm{~m}$ aguas arriba del eje del estribo. La información que de la misma surge confirma el hecho que, en la medida que la protección se torna más larga, la zona erosionada avanza hacia aguas arriba, afectando zonas no comprometidas en el ensayo de referencia. Un comportamiento semejante fue observado en el perfil ubicado $0.75 \mathrm{~m}$ aguas abajo del eje del estribo.
En la Figura 13 se muestran las erosiones resultantes en el ensayo de referencia y en los $E_{S 2-1}$ a $E_{S 2-4}$, para la sección transversal ubicada en el eje del estribo. En la misma se observa que la geometría del sector erosionado para todos los experimentos analizados que contaron con protección del lecho fue semejante, geometría que a su vez resultó similar a la que se midió en el $E_{R}$, aunque desplazada en sentido opuesto al estribo (Figura 13).

\section{Incidencia de la presencia de una protección larga sobre la configuración del flujo}

Las evidencias morfológicas recopiladas de la serie de ensayos de la Etapa I, en cuanto a la elongación de las hoyas de erosión y el apartamiento de la forma típica correspondiente a los fenómenos de erosión junto a estribos, sugirieron que la presencia de protecciones largas $\left(W_{2}\right.$ relativamente grande) junto a un estribo podría inducir que, a los mecanismos tridimensionales habituales en las proximidades de un estribo se les superpongan fenómenos típicos de contracción del flujo, cuya incidencia resulta creciente en la dirección hacia aguas abajo del mismo. 


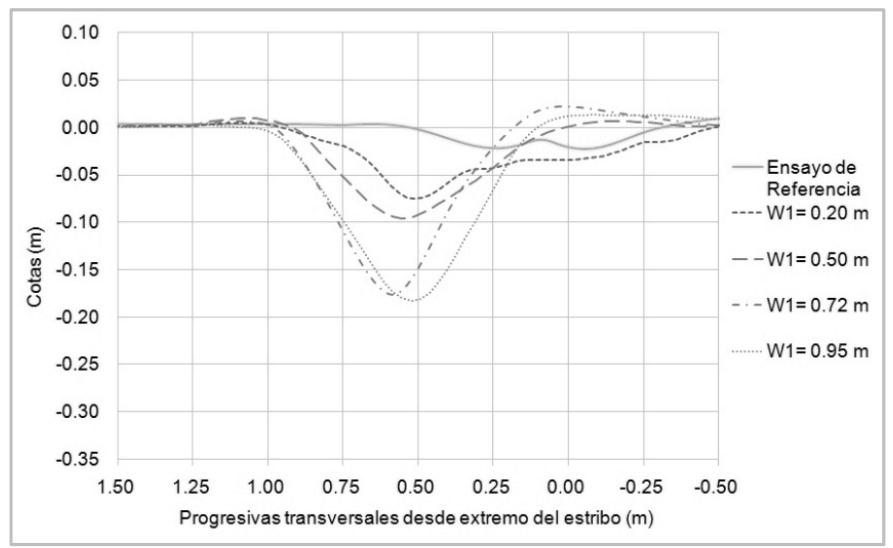

Figura 12. Perfil transversal ubicado $0,50 \mathrm{~m}$ aguas arriba del eje del Estribo

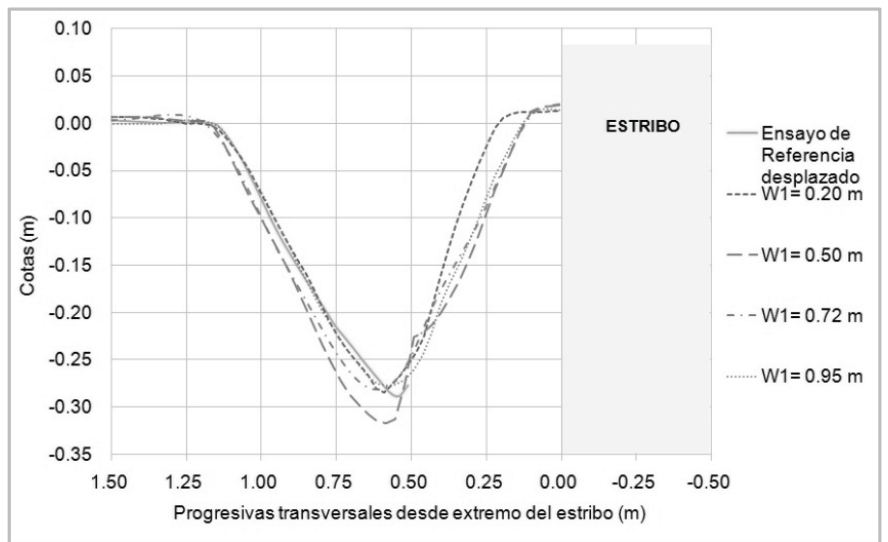

Figura 13. Perfil transversal ubicado en el eje del Estribo

Validar esta hipótesis aconsejó llevar a cabo un experimento adicional, con características particulares, cuya implementación fue descrita en el diseño experimental correspondiente a la Etapa II.

El objetivo de esta etapa fue analizar la incidencia que protecciones de gran desarrollo longitudinal podría tener sobre la configuración del escurrimiento en las inmediaciones de la misma y su interrelación con los procesos erosivos asociados.

En este sentido el estudio enfocó tres aspectos del proceso:

- Morfología del sector erosionado.

- Configuración del flujo en las inmediaciones de la protección.

- Capacidad de metodologías de estimación de erosiones desarrolladas en condiciones de flujo bidimensional de reproducir las socavaciones medidas.

\section{Morfología del sector erosionado}

En la Figura 14 se muestran cinco (5) secciones transversales relevadas al cabo de los 5 días que duró el experimento. Una de ellas se ubicó en forma coincidente con la cara de aguas arriba del estribo $\left(S_{0}\right)$ y las restantes a $0.10 \mathrm{~m} ; 0.33 \mathrm{~m}$; $0.60 \mathrm{~m}$ y $0.90 \mathrm{~m}$ aguas abajo del mismo $\left(S_{0,10}\right.$, $S_{0,33}, S_{0,60}$ y $S_{0,90}$ ). Dicha figura demuestra que tanto S0 como S10 (perfiles más próximos al estribo) presentan sus geometrías compatibles con la típica observadas en los procesos de erosión local, al tiempo que sus máximas profundidades de erosión coinciden. La sección ubicada a $0.33 \mathrm{~m}$ del estribo, S33, presenta una profundidad máxima levemente menor a las anteriores y el talud exterior del sector erosionado ligeramente más tendido, si bien la forma general de la misma coincide con la de $S_{0}$ y $S_{0,10}$. 


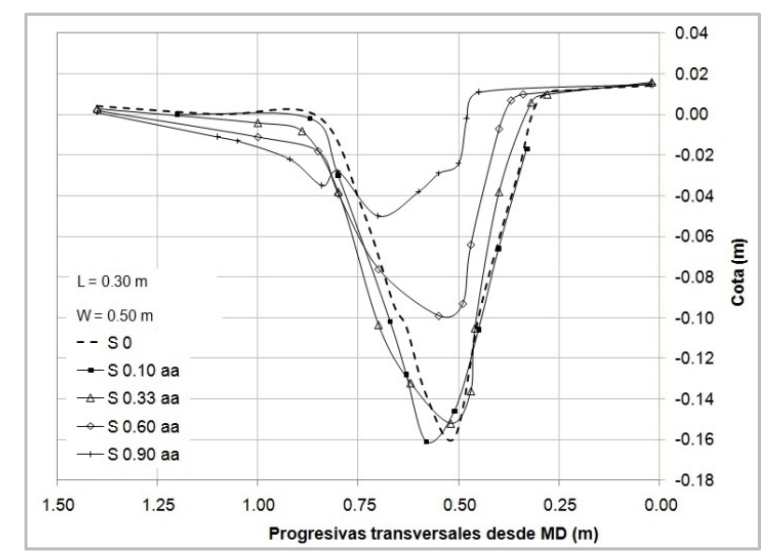

Figura 14. Perfiles transversales a lo largo de la protección. Relevamiento día 5

De la observación de la Figura 14 podría plantearse que el efecto que la configuración 3D del flujo tiene sobre la morfología del lecho resultante parece disminuir al llegar a $S_{0,60}$. En efecto, en los perfiles más distantes del estribo se observa que las máximas profundidades son sustancialmente menores y los taludes del sector erosionado de margen izquierda notoriamente mas tendidos. Particularmente en la sección $S_{0,90}$ la forma del perfil deja de ser típicamente en " $V$ " para adoptar una forma tipo " $U$ " con una franja o canal de máxima profundidad, pero con una magnitud sensiblemente menor a las de las secciones más próximas al estribo.

Las morfologías resultantes refuerzan las evidencias obtenidas en el canal de la Nave I, en cuanto a la posible acción conjunta de estructuras tridimensionales y bidimensionales responsables de las erosiones resultantes. En este marco, las erosiones observadas en la Figura 14 estarían marcando un proceso fundamentalmente tridimensional en las inmediaciones del estribo. En la medida que se consideran secciones alejadas, hacia aguas abajo del mismo, junto a la zona protegida predominan los fenómenos bidimensionales.

\section{Configuración del flujo}

Las determinaciones de velocidad efectuadas al cabo de 48 hs de iniciado el experimento, en presencia de un importante desarrollo del proceso erosivo, permitieron evaluar las distribuciones transversales de caudales para distintas secciones ubicadas a lo largo de la protección. Los valores medidos se contrastaron con la distribución de caudales obtenidos a partir de la ecuación de
Manning

$$
\% Q_{i}=\frac{(1 / n) I h_{i}^{5 / 3} \Delta B_{i}}{\sum\left((1 / n) I h_{i}^{5 / 3} \Delta B_{i}\right)}
$$

donde $\% Q i$ es el porcentaje de caudal que escurre por una dada franja " $i$ " de la sección transversal, $n$ el coeficiente de rugosidad de Manning, I la pendiente hidráulica, $h_{i}$ la profundidad media en la sección "i" y $\Delta B_{i}$ el ancho de la franja considerada.

La expresión anterior resulta aplicable a condiciones de flujo medio bidimensional, por lo que se ha considerado que su grado de ajuste a las distribuciones de caudales medidas en las distintas secciones relevadas, resultará un indicador válido de las condiciones de tridimensionalidad o bidimensionalidad del flujo (3D ó 2D) imperantes en cada una de esas secciones. La Figura 15 demuestra lo anteriormente señalado. En efecto, la distribución de caudales medidos en la sección del estribo $S_{0}$ (Figura 15) difiere de los resultados obtenidos a partir de Manning, poniendo de manifiesto la incapacidad del método para representar la compleja configuración del flujo en el sector próximo al estribo, determinado por un escurrimiento de neto corte 3D.

En la medida que se analizaron secciones transversales cada vez más distantes del estribo, en la dirección hacia aguas abajo, las distribuciones de caudales calculadas fueron aproximándose a las medidas, poniendo en evidencia a la morfología de las secciones transversales, determinantes para el cálculo de la distribución por Manning, como factor preponderante en la configuración del flujo. Este hecho alcanza su mayor dimensión en la sección ubicada $0.74 \mathrm{~cm}$ aguas abajo del estribo $\left(S_{0,74}\right)$, donde ambas distribuciones prácticamente coinciden (Figura 16). 


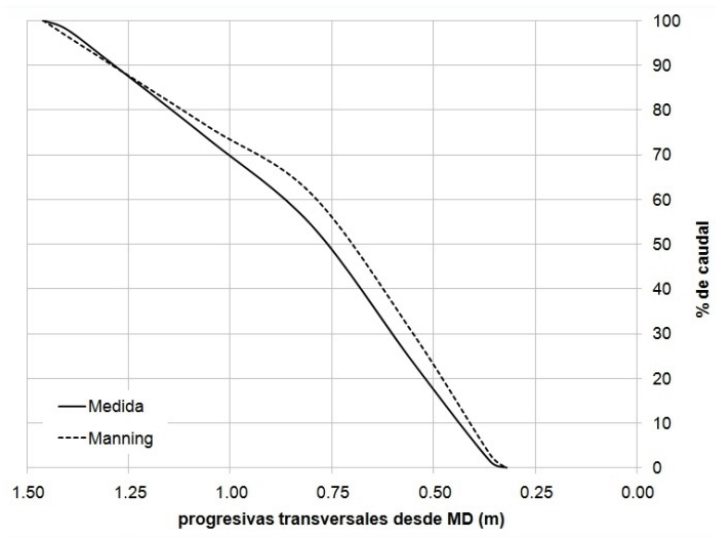

Figura 15. Comparación $\% Q$ medido - calculado. Sección $S_{0}$

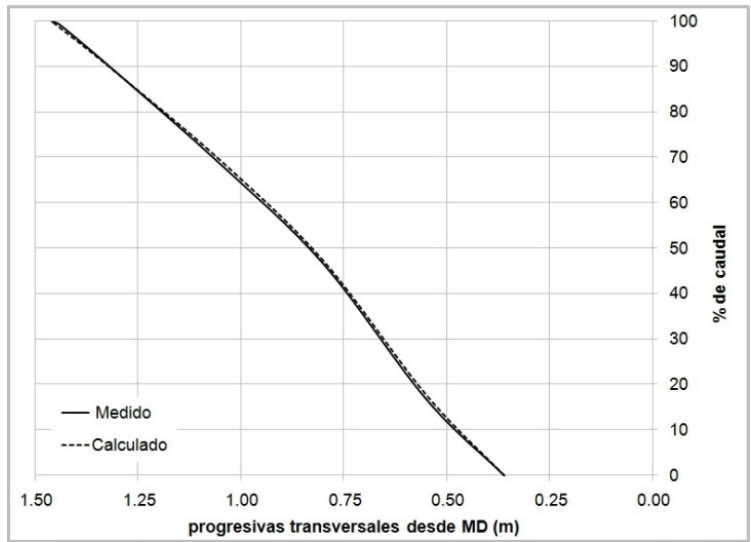

Figura 16. Comparación $\% Q$ medido - calculado. Sección $S_{0,74}$ aa

\section{Capacidad de metodologías empíricas dispo- nibles para reproducir las erosiones medidas en las inmediaciones de la protección}

Del mismo modo que la distribución calculada por Manning mostró un comportamiento desigual según la sección transversal donde se aplicó, el hecho de utilizar una fórmula de estimación de erosiones desarrolladas en condiciones de bidimensionalidad debiera manifestar un comportamiento similar.

Para demostrar esta premisa se recurrió a la metodología propuesta por Lischtvan y Lebediev, en la forma presentada por Schreider et al. (2001), ya que ha demostrado que ajusta muy bien en condiciones de flujo uniforme y bidimensional, en situaciones de escurrimiento con agua clara y profundidades menores al metro

$$
h_{s i}=\left(\frac{q_{i}}{4,7 d_{m}^{0,28} \beta}\right)^{1 / x+1}
$$

donde $h_{s i}$ es la profundidad de equilibrio en una dada vertical " $i$ ", $q_{i}$ el caudal específico actuante en la vertical "i", $d_{m}$ el diámetro característico del material del lecho, $\beta$ el coeficiente que tiene en cuenta la recurrencia del evento y $x$ el coeficiente que depende del material del lecho. Para este trabajo $x=0.41$.

La comparación de los perfiles calculados mediante (2) con los medidos al cabo de los 5 días que duró el ensayo muestra un comportamiento análogo al observado con respecto de la distribución transversal de caudales. La Figura 17 pone de manifiesto cómo la metodología empleada subestimó los valores medidos en la sección $S_{0}$, coincidente con la cara de aguas arriba del estribo. Dicha subestimación resulta razonable y previsible dado que la fórmula (2) no contempla la existencia de las estructuras de vórtices que caracterizan al flujo en dicha sección, aún en presencia de la protección. 


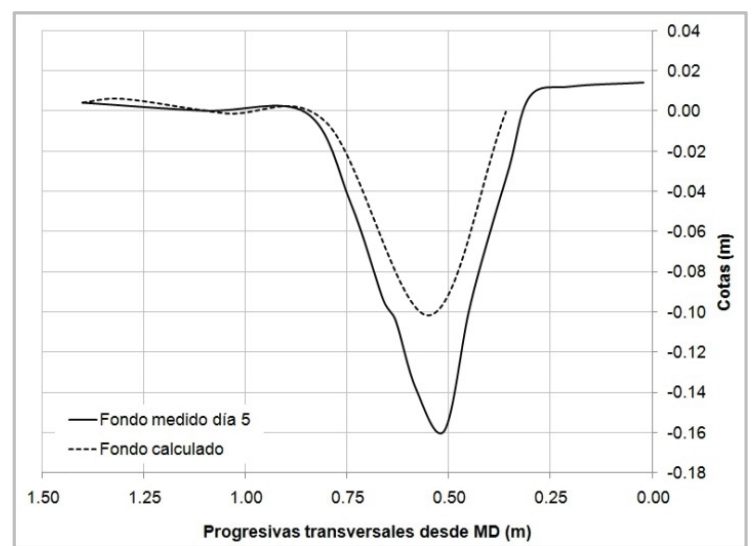

Figura 17. Perfiles de erosión medido y calculado. Sección $S_{0}$

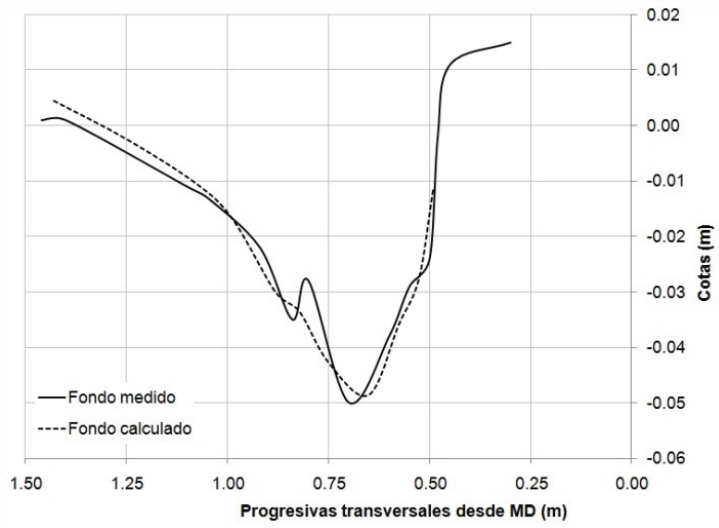

Figura 18. Perfiles de erosión medido y calculado. Sección 0.90 aguas abajo

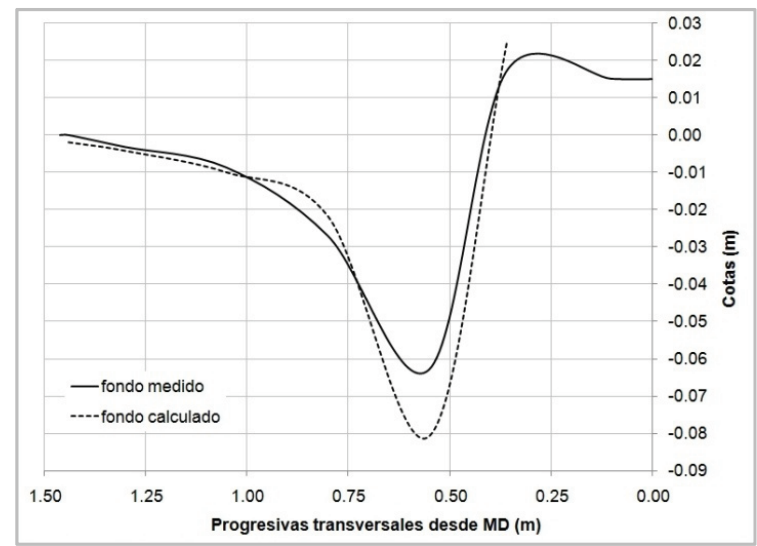

Figura 19. Perfiles de erosión medido y calculado. Sección 0.74 m aguas abajo

Por su parte, la Figura 18 muestra cómo cuando la expresión (2) se aplicó a una sección ubicada $0.90 \mathrm{~m}$ aguas abajo del estribo $\left(S_{0,90}\right)$, la reproducción del fondo medido es muy buena, poniendo de manifiesto que los efectos erosivos que se verifican en dicha sección ya no obedecen a fenómenos locales, producto de la presencia del estribo, sino al aumento de la capacidad de transporte de la corriente originada por la contracción ordenada del flujo, en lo que la protección actuaría como margen guía.

La observación del perfil $S_{0,74}$ en la Figura 19, 
ubicado en un punto intermedio, muestra cómo este proceso es gradual, pasando de una condición preponderantemente tridimensional en la sección del estribo, a un flujo típicamente bidimensional en la sección ubicada $0,90 \mathrm{~m}$ aguas abajo del sitio de emplazamiento del mismo.

\section{CONCLUSIONES}

Los resultados alcanzados muestran que las profundidades máximas de erosión obtenidas para distintas longitudes de protección fueron semejantes y muy próximas al valor testigo del ensayo $E_{R}$. En todos los casos la máxima profundidad se ubicó en las inmediaciones del eje del estribo, desplazada hacia el centro de la sección, aproximadamente $0.60 \mathrm{~m}$, respecto de su posición para la situación sin protección.

El incremento de la longitud de la protección genera una elongación de las hoyas de erosión que tuvieron lugar junto al extremo del revestimiento. En todos los casos, el desarrollo longitudinal del sector erosionado acompañó a la longitud dada a la protección, sin cambios significativos en el ancho de hoya correspondiente.

El avance hacia aguas arriba del sector erosionado, en la medida que la protección fue más larga, generó la afectación de zonas no comprometidas en la situación sin protección.

Cuando la protección fue muy corta, en el sentido longitudinal de la corriente, la zona erosionada cambió su geometría. La hoya se localizó alrededor del estribo y de la protección. La presencia del revestimiento generó una obstrucción parcial adicional al escurrimiento, actuando a modo de un estribo sumergido.

En la medida que la longitud de la protección se extendió en forma significativa hacia aguas abajo del estribo, su presencia modificó la típica configuración 3D del flujo junto al estribo, generando una superposición de mecanismos de erosión. En efecto, a los mecanismos tridimensionales habituales en las proximidades del estribo (extremo de aguas arriba de la protección) se les superponen fenómenos típicos de contracción del flujo, cuya incidencia resulta creciente en la dirección hacia aguas abajo del mismo (sector con presencia de protección). Es así que un proceso fundamentalmente tridimensional en las inmediaciones del estribo, en la medida que nos alejamos, hacia aguas abajo de la zona protegida, se transforma en bidimensional.

\section{AGRADECIMIENTOS}

A la Universidad Nacional del Litoral quien fi- nanció, por medio de los Cursos de Acción para la Investigación (CAI+D), el Proyecto de Investigación en el marco del cual se desarrolló este trabajo.

A la empresa CORIPA S.A. por el suministro de las mantas de bloques de concreto, confeccionadas especialmente para este dispositivo experimental.

Al Sr. Sebastián Schmith por su apoyo técnico en la puesta a punto de los experimentos.

\section{REFERENCIAS}

Cardoso, A.H y Fael, C., (2009). Protecting vertical-wall abutments with riprap mattresses. Journal of Hydraulic Engineering. A.S.C.E., 135(6), 457-465.

Chiew, Y.M., (1995). Mechanics of riprap failure at bridge piers'. Journal of Hydraulic Engineering, A.S.C.E., 121(9), 635-643.

Eve, N.J. y Melville, B.W., (2000). Riprap protection of abutment under clear water conditions. Part of Building Partnerships 2000 Joint Conference on Water Resource Engineering and Water Resources Planning \& Management, Section 45, Chapter 1.

García, M.H, (2008). Sedimentation engineering: processes, measurements, modeling and practice. A.S.C.E. Manuals and Reports on Engineering Practice No. 110.

Lagasse, P.F., Zevenbergen, L.W., Schall, J.D. y Clopper, P.E., (2001). Bridge scour and stream instability countermeasures. Experience, selection and design guidance. Report No. FHWA-NHI 01-003, Second Edition, Hydraulic Engineering Circular No. 23.

Lauchlan, C.S. y Melville, B.W., (2001). Riprap protection at bridge piers. Journal of $\mathrm{Hy}-$ draulic Engineering, A.S.C.E., 127(5).

May, R.W.P, Ackers, J.C. y Kirby, A.M., (2002). Manual on scour al bridges and other hydraulic structures. CIRIA C551, London, United Kingdom.

Melville, B.W. y Coleman, S.E., (2000). Bridge scour. Water Resources Publications, LLC.

Melville, B.W, van Ballegooy, S., Coleman, S.E. y Barkdoll, B., (2006). Countermeasure toe protection at spill-through abutments. Journal of Hydraulic Engineering, A.S.C.E. 132(3), 235-245. 
Morales, R., Ettema, R. y Barkdoll, B. (2008). Large-scale flume tests of riprap-apron performance at a bridge abutment on a floodplain. Journal of Hydraulic Engineering. A.S.C.E., 134(6), 800-809.

Pilarczyk, K.W., (1997). Simplified unification of stability formulae for revetment. Chapter 4, in River, Coastal and Shoreline Protection, C.R. Thorne, S.R. Abt, F.B.J. Barends, S.T. Maynord y K.W. Pilarczyk (Eds.), John Wiley \& Sons, Inc. New York, USA.

Przedwojski, R., Blazejewski, R. y Pilarczyk, K.W., (1995). River training techniques, fundamentals, design and applications. A.A. Balkema, Rotterdam.

Reynares, M.L., Roca Collell, M., Scacchi, G.B. y Schreider, M.I., (2005). Incidencia de las protecciones de estribo en el desarrollo de la erosión local. XX Congreso Nacional del Agua, Mendoza, Argentina.

Richardson, E.V. y Davis, S.R., (1995). Evaluating scour at bridges. Report No FHW-
IP-90-017, Hydraulics Engineering Circular No 18 (HEC 18), Third Edition, Office of Technology Applications, HIA-22, Federal Highway Administration, U.S.

Scacchi, G., Schreider, M. y Fuentes, R., (2005). Erosión en estribos de puentes aliviadores ubicados en valles de inundación. Revista Ingeniería Hidráulica en México, Vol XX (3), 43-59.

Schreider, M., Scacchi, G. y Franco, F., (2001). Aplicación del método de Lischtvan y Lebediev al cálculo de la erosión general. Revista Ingeniería Hidráulica en México, XVI (1), 15-26.

Schreider, M.. Zanardi, L., Scacchi, G. y Franco, F., (1998). Erosión por contracción y por estribo en puentes aliviadores en valle de inundación. Revista Ingeniería del Agua, 5(2), 23-34.

Témez, J.R., (1988). Control de la erosión fluvial en puentes. Ministerio de Obras Públicas y Urbanismo (MOPU), Madrid, España. 